\title{
Chern Numbers of Algebraic Surfaces
}

\section{An Example}

\section{F. Hirzebruch}

Max-Planck-Institut für Mathematik, Gottfried-Claren-Strasse 26, D-5300 Bonn 3, Federal Republic of Germany

Max Koecher zum 60. Geburtstag gewidmet

An algebraic surface has the Chern numbers $c_{2}$ (Euler-Poincaré characteristic or Euler number) and $c_{1}^{2}$ (selfintersection number of a canonical divisor). For a minimal surface of general type these numbers are positive and satisfy

$$
c_{1}^{2} \leqq 3 c_{2} \quad(\text { see }[4,6]) .
$$

If $\left\{E_{i}\right\}$ is a finite set of disjoint smooth elliptic curves on a minimal surface of general type, then $E_{i} E_{i}<0$ and

$$
-\sum E_{i} E_{i} \leqq 3 c_{2}-c_{1}^{2} \quad(\text { see }[5, \text { Sect. 7]) }
$$

We shall construct a sequence $X_{n}(n=2,3, \ldots)$ of minimal surfaces of general type with the following properties

$$
\begin{gathered}
c_{2}\left(X_{n}\right)=n^{7} \\
3 c_{2}\left(X_{n}\right)-c_{1}^{2}\left(X_{n}\right)=4 n^{5} .
\end{gathered}
$$

There are $4 n^{4}$ smooth disjoint elliptic curves on $X_{n}$ with selfintersection number $-n$, showing that we have the equality sign in (2). As a consequence of (3) and (4) we have

but

$$
c_{1}^{2}\left(X_{n}\right) / c_{2}\left(X_{n}\right)<3
$$

$$
\lim _{n \rightarrow \infty} c_{1}^{2}\left(X_{n}\right) / c_{2}\left(X_{n}\right)=3
$$

Holzapfel $[2,3]$ has made a thorough investigation of Picard modular surfaces as quotients of the complex ball. He showed that the Picard modular surfaces give examples of (2) with equality sign where the $E_{i}$ correspond to the resolution of the cusps. Holzapfel observed that the Picard modular surfaces give many examples of surfaces with $c_{1}^{2} / c_{2}$ arbitrarily near to 3 . But it may be of some interest to give a direct geometric construction of surfaces $X_{n}$ satisfying (5). Perhaps the $X_{n}$ we are going to construct are Picard modular surfaces. In any case, it should be conjectured that the universal cover of $X_{n}-\bigcup E_{i}$ is the ball. For more references to the literature see [1]. Anyhow, this note should be regarded as a continuation of [1]. 


\section{Abelian Coverings of an Abelian Surface}

Let $\zeta=e^{2 \pi i / 6}$ and $T$ the elliptic curve

$$
T=\mathbb{C} / \mathbb{Z} \cdot 1+\mathbb{Z} \zeta .
$$

Consider the abelian surface $T \times T$ whose points are denoted by $(z, w)$. The curve $T$ has complex multiplication by the algebraic integers $\alpha \in \mathbb{Q}[\zeta]$ and $w=\alpha z$ is an elliptic curve $T_{\alpha}$ in $T \times T$ isomorphic to $T$. We take the following curves on $T \times T$

$$
\begin{gathered}
T_{0}: w=0 \\
T_{\infty}: z=0 \\
T_{1}: w=z \\
T_{\zeta}: w=\zeta z .
\end{gathered}
$$

The four curves pass through the origin of $T \times T$ and do not intersect anywhere else. It suffices to remark that $T_{1} \cap T_{\zeta}=\{(0,0)\}$. This follows from the fact that $1-\zeta$ is a unit, namely $1-\zeta=-\zeta^{2}$.

Let $U_{n}$ be the group of $n$-division points of $T \times T$,

$$
U_{n}=\{(z, w) \mid(n z, n w)=(0,0)\} .
$$

It has order $n^{4}$. The group $U_{n}$ acts on $T \times T$ by translations, each of the four curves remaining invariant under a group of $n^{2}$ translations contained in $U_{n}$. Therefore $U_{n}\left(T_{0}\right), U_{n}\left(T_{\infty}\right), U_{n}\left(T_{1}\right), U_{n}\left(T_{\tau}\right)$ each consists of $n^{2}$ smooth disjoint elliptic curves. Altogether we have $4 n^{2}$ elliptic curves. Through each of the $n^{4}$ points of $U_{n}$ (considered as subset of $T \times T)$ pass 4 of the $4 n^{2}$ curves. There are no other intersections.

We introduce the divisors

$$
D_{0}=U_{n}\left(T_{0}\right), \quad D_{\infty}=U_{n}\left(T_{\infty}\right), \quad D_{1}=U_{n}\left(T_{1}\right), \quad D_{\zeta}=U_{n}\left(T_{\xi}\right)
$$

on $T \times T$. Each such divisor is a sum of $n^{2}$ disjoint smooth curves (of multiplicity 1). In the integral homology group $H_{2}(T \times T, \mathbb{Z})$ each $D_{i}(i \in\{0, \infty, 1, \zeta\})$ is divisible by $n^{2}$, since $u T_{i}$ and $T_{i}$ determine the same homology class if $u$ is a translation. In fact,

$$
D_{i} \approx n^{2} T_{i}
$$

where $\approx$ denotes linear equivalence. This is clear, because $T_{i}$ is a direct factor of $T \times T$ and since on an elliptic curve $n^{2}(0)$ is linearly equivalent to the divisor consisting of the $n^{2}$ division points of order $n$. Let $f_{i}$ be the meromorphic function on $T \times T$ whose divisor equals $D_{i}-n^{2} T_{i}$. It is uniquely determined up to a constant factor. Observe that the divisor of $f_{i} / f_{j}$ restricted to a component of $D_{k}$ (where $i, j . k$ are distinct elements of $\{0, \infty, 1, \zeta\})$ is of the form $n^{2}(a)-n^{2}(b)$ where $a, b$ are points of the component such that $a-b$ is an $n$-division point in the underlying group structure. Therefore $n(a) \approx n(b)$.

Thus $f_{i} / f_{j}$ restricted to a component of $D_{k}$ becomes an $n^{\text {th }}$ power of a meromorphic function.

Using the $f_{i}$ we construct the algebraic surface $X_{n}$. 
The function field of $X_{n}$ will be the function field of $T \times T$ with $\left(f_{1} / f_{0}\right)^{1 / n}$, $\left(f_{o s} / f_{0}\right)^{1 / n},\left(f_{t} / f_{0}\right)^{1 / n}$ adjoined to it. This already defines the birational equivalence class of $X_{n}$, To get a smooth model we blow up the $n^{4}$ points of $T \times T$ belonging to $U_{n}$. We get an algebraic surface $Y_{n}$ with Euler number $n^{4}$ and $n^{4}$ exceptional curves $L_{j}\left(j \in U_{n}\right)$ arising from blowing up. The strict transforms of the $D_{i}$ are smooth curves $\hat{D}_{i}$ in $Y_{n}$ which do not intersect each other and $\left(f_{1} / f_{0}\right)^{1 / n},\left(f_{\infty} / f_{0}\right)^{1 / n},\left(f_{5} / f_{0}\right)^{1 / n}$ determine a smooth covering $X_{n}$ of $Y_{n}$ of degree $n^{3}$ ramified above the $\tilde{D}_{i}$ with ramification index $n$. Since the branching locus consists of elliptic curves which have Euler number 0, the Hurwitz method for calculating Euler numbers gives

$$
c_{2}\left(X_{n}\right)=n^{3} c_{2}\left(Y_{n}\right)=n^{3} \cdot n^{4}=n^{7} \text {. }
$$

Let $\pi: X_{n} \rightarrow Y_{n}$ be the covering map. Then

$$
\pi^{*} \tilde{D}_{k}=n \tilde{D}_{k} \quad(k \in\{0, \infty, 1, \zeta\})
$$

and $\bar{D}_{k}$ consists of $n^{4}$ disjoint elliptic curves. Over each of the $n^{2}$ components of $\tilde{D}_{k}$ lie $n^{2}$ components of $\bar{D}_{k}$. This follows from the fact mentioned above that $f_{i} / f_{j}$, restricted to a component of $\tilde{D}_{k}$, is the $n^{\text {th }}$ power of a meromorphic function. Each component of $\bar{D}_{k}$ has selfintersection number $-n$. To check this observe first that $D_{k} D_{k}=0$ and $\tilde{D}_{k} \tilde{D}_{k}=-n^{4}$, since $D_{k}$ passes through the $n^{4}$ points of $T \times T$ which were blown up. Then we have

$$
\begin{gathered}
\pi^{*} \tilde{D}_{k} \cdot \pi^{*} \tilde{D}_{k}=n^{3}\left(\tilde{D}_{k} \cdot \tilde{D}_{k}\right)=-n^{7}=\left(n \bar{D}_{k}\right) \cdot\left(n \bar{D}_{k}\right) \\
\bar{D}_{k} \bar{D}_{k}=-n^{5} \quad(k \in\{0, \infty, 1, \zeta\}) .
\end{gathered}
$$

Thus each of the $n^{4}$ components of $\bar{D}_{k}$ has selfintersection number $-n$.

For each exceptional curve $L_{j}$ of $Y_{n}$ (where $j \in U_{n}$ ) the lifted curve $\pi^{*} L_{j}$ is a smooth curve $\bar{L}_{j}$ in $X_{n}$ with Euler number

$$
e\left(\bar{L}_{j}\right)=-2 n^{3}+4 n^{2} .
$$

This follows from Hurwitz's formula since the map $\bar{L}_{j} \rightarrow L_{j}$ is of degree $n^{3}$ with ramification over 4 points of $L_{j}$ with ramification index $n$ everywhere. We have

$$
\bar{L}_{j} \bar{L}_{j}=-n^{3} \text {. }
$$

The surface $Y_{n}$ has a unique effective canonical divisor $K$, namely $K=\sum L_{j}\left(j \in U_{n}\right)$. Thus

$$
\bar{K}=\sum_{j \in U_{n}} \bar{L}_{j}+(n-1) \sum_{i \in\{0,1, \infty, \zeta\}} \bar{D}_{i}
$$

is an effective canonical divisor of $X_{n}$. For $n \geqq 2$, the divisor $\bar{K}$ does not contain any rational curves. So $X_{n}$ is free of exceptional curves, because an exceptional curve is contained in every effective canonical divisor. Hence the surface $X_{n}$ is minimal for $n \geqq 2$.

For the calculation of $c_{1}^{2}\left(X_{n}\right)=\bar{K} \bar{K}$ we have to use that

$$
\bar{L}_{j} \bar{D}_{i}=n^{2} \quad\left(j \in U_{n}, i \in\{0, \infty, 1, \zeta\}\right)
$$


which follows from the fact that over a ramification point on $L_{j}$ of $\bar{L}_{j} \rightarrow L_{j}$ lie $n^{2}$ points on $\bar{L}_{j}$. Using (7), (9), and (11) we get from (10)

$$
\begin{aligned}
c_{1}^{2}\left(X_{n}\right) & =\sum \bar{L}_{j} \bar{L}_{j}+2(n-1) \sum \bar{L}_{j} \bar{D}_{i}+(n-1)^{2} \sum \bar{D}_{i} \bar{D}_{i} \\
& =n^{4}\left(-n^{3}\right)+2(n-1) \cdot 4 n^{4} \cdot n^{2}+(n-1)^{2} \cdot 4\left(-n^{5}\right) \\
c_{1}^{2}\left(X_{n}\right) & =3 n^{7}-4 n^{5} .
\end{aligned}
$$

This number is positive for $n \geqq 2$ and thus $X_{n}$ is of general type.

We have proved all statements mentioned in the introduction. The $4 n^{4}$ elliptic curves of selfintersection number $-n$ come from the four elliptic curves $T_{0}, T_{\infty}, T_{1}$, $T_{\xi}$.

\section{A Surface with $c_{1}^{2}=3 c_{2}$}

Consider the surface $X_{n}$ constructed in Sect. 1. Adjoining to the function field of $X_{n}$ the element $\left(f_{0}\right)^{1 / n}$ [or equivalently $\left(f_{1}\right)^{1 / n},\left(f_{\infty}\right)^{1 / n},\left(f_{\xi}\right)^{1 / n}$, defines an $n$-fold covering of $X_{n}$ ramified along the curves $\bar{L}_{j}$. We obtain a smooth surface $W_{n}$. By Hurwitz's method

$$
c_{2}\left(W_{n}\right)=n c_{2}\left(X_{n}\right)-(n-1) \sum e\left(\bar{L}_{j}\right)
$$

and by (6) and (8)

$$
\begin{aligned}
& c_{2}\left(\boldsymbol{W}_{n}\right)=n^{8}-(n-1) n^{4}\left(-2 n^{3}+4 n^{2}\right), \\
& c_{2}\left(\boldsymbol{W}_{n}\right)=n^{6}\left(3 n^{2}-6 n+4\right) .
\end{aligned}
$$

The divisor $\bar{K}+\frac{n-1}{n} \sum \bar{L}_{j}$ gives, when lifted to $W_{n}$, a canonical divisor of $W_{n}$. Thus

$$
\begin{aligned}
c_{1}^{2}\left(W_{n}\right) & =n\left(\bar{K}+\frac{n-1}{n} \sum \bar{L}_{j}\right)^{2} \\
& =n\left(\bar{K}^{2}+2 \frac{n-1}{n} \sum \bar{K} \bar{L}_{j}+\frac{(n-1)^{2}}{n^{2}} \sum \bar{L}_{j}^{2}\right) .
\end{aligned}
$$

By (9)-(11) we have

$$
\bar{K} \bar{L}_{j}=3 n^{3}-4 n^{2}
$$

and by (14), (12), (15), and (9)

$$
\begin{aligned}
& c_{1}^{2}\left(W_{n}\right)=n\left(3 n^{7}-4 n^{5}+2(n-1) n^{3}\left(3 n^{3}-4 n^{2}\right)-(n-1)^{2} n^{5}\right) \\
& c_{1}^{2}\left(W_{n}\right)=n^{6}\left(8 n^{2}-12 n+3\right) .
\end{aligned}
$$

Hence by (13) and (16)

$$
3 c_{2}\left(W_{n}\right)-c_{1}^{2}\left(W_{n}\right)=n^{6}(n-3)^{2} .
$$

Thus $W_{3}$ is a surface (minimal, of general type) with $c_{1}^{2}=3 c_{2}$.

We observe that

$$
\lim _{n \rightarrow \infty} \frac{c_{1}^{2}\left(W_{n}\right)}{c_{2}\left(W_{n}\right)}=\frac{8}{3}
$$




\section{Elliptic Functions}

Consider an elliptic curve $\mathbb{C} / \mathbb{Z} \omega_{1}+\mathbb{Z} \omega_{2}$. The Weierstrass $\sigma$-function can be used to describe elliptic functions with given zeros and poles: If $a_{i}(i=1, \ldots, m)$ and $b_{i}$ $(i=1, \ldots, m)$ are complex numbers satisfying

$$
\sum a_{i}=\sum b_{i} \bmod \mathbb{Z} \omega_{1}+\mathbb{Z} \omega_{2}
$$

then

$$
\prod_{i=1}^{n}\left(\sigma-a_{i}\right) / \prod_{i=1}^{n}\left(\sigma-b_{i}\right)
$$

is an elliptic function with zeros and poles in $a_{i}$ and $b_{i}$, respectively. Its divisor equals

$$
\sum\left(a_{i}\right)-\sum\left(b_{i}\right)
$$

where now addition and subtraction are in the group of divisors of the curve. In particular, we can define the elliptic function

$$
F_{n}(z)=\prod_{a \in U_{n}} \sigma(z-a) / \sigma(z)^{n^{2}}=\text { const } \cdot \sigma(n z) / \sigma(z)^{n^{2}},
$$

where $U_{n}$ is the group of $n$-division points of the curve.

We now take the lattice $\mathbb{Z}+\mathbb{Z} \zeta$ of Sect. 1 , and use also the notation of Sect. 1 . Then we can write the functions $f_{0}, f_{\infty}, f_{1}, f_{\zeta}$ as follows

$$
\begin{gathered}
f_{0}=F_{n}(w), \quad f_{\infty}=F_{n}(z) \\
f_{1}=F_{n}(w-z), \quad f_{\zeta}=F_{n}(w-\zeta z) .
\end{gathered}
$$

Thus the function field of the algebraic surface $X_{n}$ is the field of meromorphic functions of $z$ and $w$ elliptic in both variables (with respect to the lattice $\mathbb{Z}+\mathbb{Z} \zeta$ ) extended by adjoining

$$
\left(F_{n}(z) / F_{n}(w)\right)^{1 / n}, \quad\left(F_{n}(w-z) / F_{n}(w)\right)^{1 / n}, \quad\left(F_{n}(w-\zeta z) / F_{n}(w)\right)^{1 / n} .
$$

The function field of $W_{n}$ is obtained by adjoining

$$
F_{n}(z)^{1 / n}, \quad F_{n}(w)^{1 / n}, \quad F_{n}(w-z)^{1 / n}, \quad F_{n}(w-\zeta z)^{1 / n} .
$$

Remark. I came to the constructions of Sect. 1, Sect. 2 by trying to replace line arrangements (see [1]) by other interesting arrangements of curves on some surface. Then I realized how close the constructions of Sect. 1, Sect. 2 are to the paper of Livné (Harvard thesis, see [1]). He takes the elliptic modular surface $E(n)$ of level $n$ and uses the $n^{2}$ sections of the elliptic fibration $E(n) \rightarrow X(n)$ as locus of ramification. I write $T \times T$ in different ways as direct product and thus as a trivial fibration and use its $n^{2}$ sections of $n$-division points as locus of ramification, applying several such ramifications simultaneously.

The methods of Sect. 1, Sect. 2 lead to interesting surfaces also for other elliptic curves with complex multiplication. The special fact occuring for the curve $T$ is that the four elliptic curves $T_{0}, T_{\infty}, T_{1}, T_{\zeta}$ meet only in the origin of $T \times T$. 
Acknowledgements. This paper was written in March 1983 when I was visiting the Mathematical Sciences Research Institute in Berkeley. I would like to thank the MSRI for the invitation and the hospitality. During my visit of the MSRI I met S-T. Yau who told me that he has proved his uniformization theorem also in the relative (logarithmic) case: The equality sign in (2) implies that the universal cover of the surface with the union of the elliptic curves removed is the ball.

\section{References}

1. Hirzebruch, F.: Arrangements of lines and algebraic surfaces. In: Volume dedicated to I.R. Shafarevich. Progress in Mathematics, Vol. 36, pp. 113-140. Boston: Birkhäuser 1983

2. Holzapfel, R.P.: A class of minimal surfaces in the unknown region of surface geography. Math. Nachr. 98, 211-232 (1980)

3. Holzapfel, R.P.: Invariants of arithmetical ball quotient surfaces. Math. Nachr. 103, 117-153 (1981)

4. Miyaoka, Y.: On the Chern numbers of surfaces of general type. Invent. Math. 42, 225-237 (1977)

5. Sakai, F.: Semi-stable curves on algebraic surfaces and logarithmic pluricanonical maps. Math. Ann. 254, 89-120 (1980)

6. Yau, S.-T.: Calabi's conjecture and some new results in algebraic geometry. Proc. Nat. Acad. Sci. USA 74, 1798-1799 (1977)

Received April 28, 1983 